\title{
A complicated case of primary hypophysitis with bilateral intracavernous carotid artery occlusion
}

\author{
Pinelopi Katsiveli, ${ }^{1}$ Maria Sfakiotaki, ${ }^{1}$ Nikolaos Voulgaris, ${ }^{1}$ Labrini Papanastasiou, ${ }^{1}$ \\ Theodora Kounadi, ${ }^{1}$ Konstantinos Lymperopoulos, ${ }^{2}$ George Piaditis'
}

${ }^{1}$ Department of Endocrinology and Diabetes Center, ${ }^{2}$ Department of Interventional Radiology, General Hospital of Athens "G. Gennimatas", Athens, Greece

\begin{abstract}
Primary hypophysitis (PH) is a rare clinical entity characterized by inflammatory infiltration of the pituitary gland with various degrees of pituitary dysfunction. OBJECTIVE: To present a complicated case of aggressive $P H$ with bilateral cavernous sinuses infiltration, successfully treated with azathioprine after failure of corticosteroid treatment. METHODS AND RESULTS: A 48-year-old woman presented with episodes of recurrent headache and progressively worsening muscle weakness. Magnetic resonance imaging (MRI) identified an intrasellar pituitary lesion with thickened pituitary stalk extending to the cavernous sinuses and causing asymptomatic occlusion of both internal carotid arteries (ICAs). Hormonal investigation showed severe anterior pituitary deficiency. The diagnosis of $\mathrm{PH}$, and more specifically of lymphocytic hypophysitis (LYH), was suspected and glucocorticoid treatment was initiated. Because of the patient's susceptibility to infections, the attempt to gradually reduce glucocorticoid dosage induced a relapse of PH. Immunosuppressive therapy with azathioprine was administered. Significant pituitary mass reduction with regression of the inflammation to the cavernous sinuses was documented. At follow-up the pituitary function was normal, while the patient was on the minimum dose of azathioprine. Thereafter, azathioprine was discontinued without any clinical/biochemical or radiological evidence of PH except for the permanent ICA occlusion. CONCLUSIONS: Despite its rarity, PH should be included in the differential diagnosis of pituitary masses and involvement of ICAs occlusion should not be underestimated. Azathioprine, applied as an alternative treatment, was shown to result in remarkable $\mathrm{PH}$ improvement.
\end{abstract}

Key words: Azathioprine, Carotid artery occlusion, Lymphocytic hypophysitis, Primary hypophysitis

Address for correspondence:

Labrini Papanastasiou, Department of Endocrinology and

Diabetes Center, Athens General Hospital "G. Gennimatas",

154 Mesogion Avenue, 11527 Athens, Greece;

Tel.: +30 2107768283, Fax: +30 2107779146,

E-mail: linapapan@yahoo.gr

Received: 18-12-2015, Accepted: 22-03-2016

\section{INTRODUCTION}

Primary hypophysitis $(\mathrm{PH})$ is a rarely recognized disorder, characterized by localized or generalized inflammatory infiltration of the pituitary gland with various degrees of pituitary dysfunction. It can manifest as a sellar mass, which may be difficult to differenti- 
ate from other space-occupying lesions. Although the aetiology of the disease has not yet been fully elucidated, an autoimmune mechanism is most likely implicated. Three histopathologic subtypes are recognized under the term of PH: lymphocytic (LYH), granulomatous $(\mathrm{GH})$ and xanthomatous hypophysitis $(\mathrm{XH})$. According to other investigators, xanthogranulomatous and necrotizing hypophysitis should also be added to the classification of PH. ${ }^{1-3}$ The clinical and hormonal manifestations, similar to all classifications, include symptoms of sellar compression (headache, visual field impairment) and of anterior and posterior pituitary deficiency. MRI can be particularly important in differentiating $\mathrm{PH}$ from an adenoma; intrasellar and suprasellar pituitary enlargement with thickened pituitary stalk is the most common imaging characteristic, especially when diabetes insipidus is present. The final diagnosis of $\mathrm{PH}$ is made via biopsy and subsequent histopathology, although antipituitary antibodies (APA) detection is increasingly being used in order to differentiate $\mathrm{PH}$ from pituitary adenoma. Histological examination of the adenohypophysis shows a lymphocytic infiltration, which is to a variable degree associated with destruction and fibrosis of the parenchyma. ${ }^{4}$ Glucocorticoids are considered as the treatment of choice, while use of immunosuppressive drugs, such as azathioprine, has proved to be effective and could represent a viable alternative treatment. ${ }^{5-7}$ A more aggressive surgical approach or stereotactic radiotherapy should be applied in the subset of patients unresponsive to medical therapy or with compressive symptoms. ${ }^{8,9}$

Bilateral internal carotid arteries (ICAs) occlusion associated with $\mathrm{PH}$ is an exceedingly rare clinical entity, the occlusion apparently arising from inflammation spreading to the walls of the vessels.

The aim of this article is to present a particularly unusual case of $\mathrm{PH}$ with bilateral cavernous sinuses infiltration and bilateral ICAs occlusion, successfully treated with azathioprine after failure of corticosteroids.

\section{CASE REPORT}

A 48-year-old woman was referred to our Department for evaluation of persisting headache progressively worsening over the previous months and for muscle weakness during the same period. She is a mother of two children_and went through her menopause at the age of 44 years. On admission, clinical examination was unremarkable (blood pressure $110 / 70 \mathrm{mmHg}$, pulse rate $70 / \mathrm{min}$ and body temperature $36.6^{\circ} \mathrm{C}$ ) and there was no clinical evidence of autoimmune disease. MRI of the pituitary gland showed an intrasellar $1.5 \times 1.5 \mathrm{~cm}$ pituitary lesion extending slightly into the supersellar area, in close relation to the optic chiasm, invading the cavernous sinuses and causing asymptomatic occlusion of both internal carotid arteries. Normal flow signal in both ICAs was absent. Furthermore, a thickened pituitary stalk was observed (Figure 1). The patient's visual fields test was normal. Although the pituitary lesion invaded the cavernous sinuses, symptoms implicating the $3^{\text {rd }}, 4^{\text {th }}, 5^{\text {th }}$ and $6^{\text {th }}$ cranial nerves -such as diplopia, strabismus and fall of the upper eyelid- were not apparent. Hormonal investigation showed complete anterior pituitary deficiency [T3: $0.49 \mathrm{ng} / \mathrm{ml}(0.58-$ 1.59) FT4: $8.12 \mathrm{pmol} / \mathrm{L}$ (9.01-21), TSH: $2 \mu \mathrm{IU} / \mathrm{ml}$ (0.35-4.94), LH: $0.53 \mathrm{mIU} / \mathrm{ml}$ (10.39-64.57), FSH: $2.77 \mathrm{mIU} / \mathrm{ml}(26.72-133.41), \mathrm{E} 2:<10 \mathrm{pmol} / \mathrm{L}(<36-$ 102), PRL: $<0.6 \mathrm{ng} / \mathrm{ml}(5.18-26.53)$, ACTH: $<5 \mathrm{pg} / \mathrm{ml}$ (9-52), Cortisol: 143 nmol/L (138-690), IGF-1: 73.9 $\mathrm{ng} / \mathrm{ml}$ (180-460), anti-TPO (negative)], whereas there was no evidence of diabetes insipidus. Consequently, the patient received hydrocortisone and thyroxine as replacement therapy.

The clinical symptoms, imaging findings and hormonal profile led to the diagnosis of an infiltrative or inflammatory lesion, such as PH. Serum and cerebrospinal fluid (CSF) angiotensin converting enzyme, VDRL testing for syphilis, PCR for tuberculosis and immunological investigation were normal. CSF cultures, including mycobacterium, were negative therefore excluding infectious or granulomatous diseases such as neurosarcoidosis, syphilis and tuberculosis. Computed tomography (CT) of the thorax and abdomen were normal.

We considered LYH as the most likely diagnosis, although there were no signs of other autoimmune diseases, such as Hashimoto's thyroiditis, which often coexist with this clinical entity. Methylprednisolone treatment was initiated (48mg daily, tapered over a 12-week period), preserving thyroxine treatment. Her headaches and weakness improved rapidly, thus a watch-and-wait approach was adopted. Concur- 


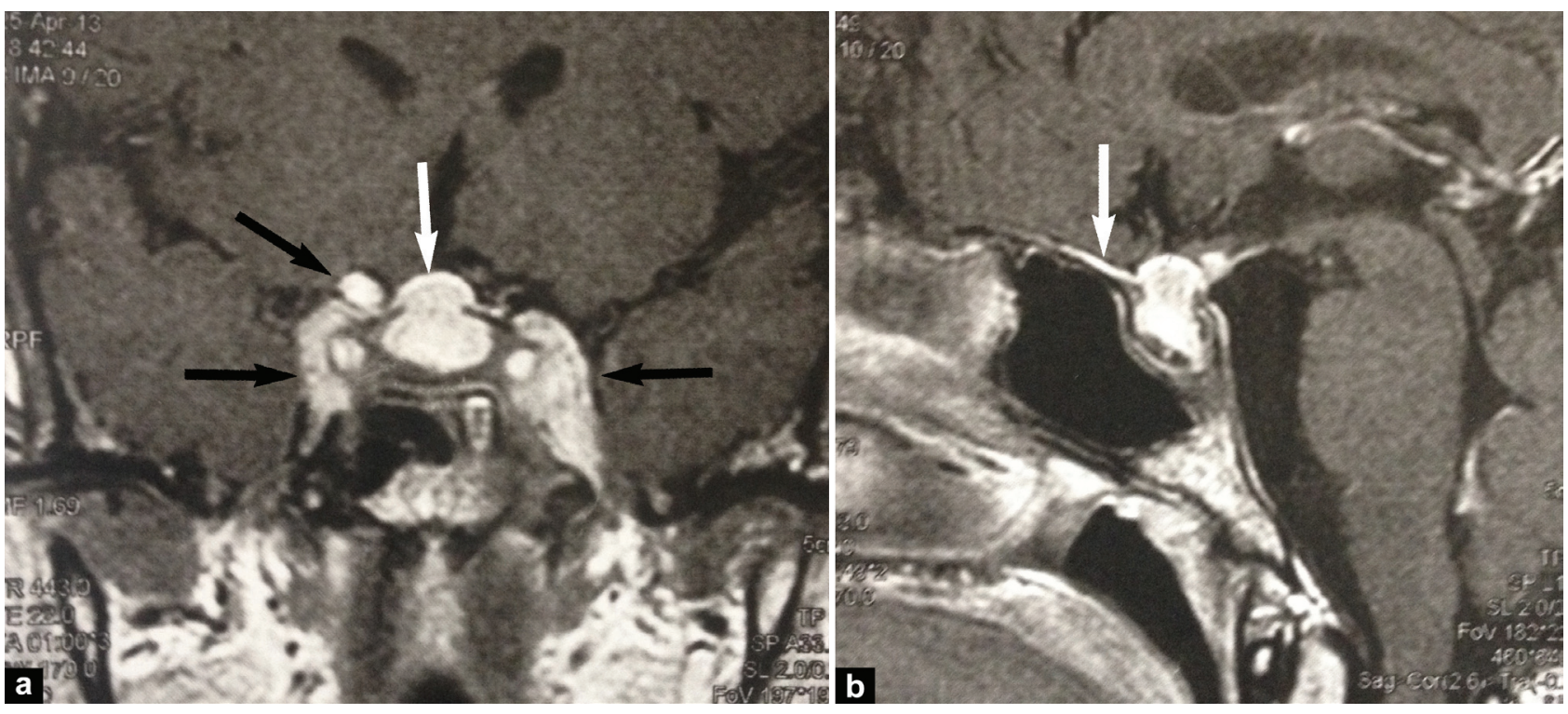

Figure 1. a) Pituitary coronal and sagittal T1-weighed MRI scan after gadolinium enhancement. The pre-treatment MRI figures depicted an intrasellar pituitary lesion invading the cavernous sinuses (white arrow). Normal flow signal in both ICAs was absent (black arrows). b) Dural tail with normal bright spot was also identified (white arrow).

rently, calcium and alendronate administration were initiated. Six months later, slurred speech and rightsided paresis developed, requiring admission to the Neurological Department. A brain MRI scan showed small frontopartial ischemic infarcts strongly suggestive of a subacute left hemisphere stroke. The size of the pituitary lesion was reduced, being less invasive of the cavernous sinuses compared to the previous imaging (Figure 2). The magnetic resonance angiog-

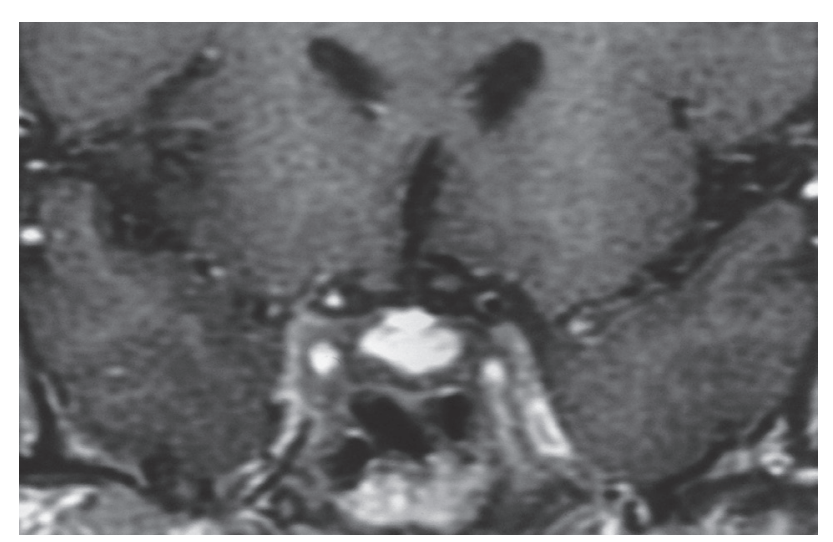

Figure 2. Pituitary coronal T1-weighed MRI scan after gadolinium enhancement six months after methylprednisolone treatment was initiated. The size of the pituitary lesion is reduced, invading less the cavernous sinuses compared to the previous imaging. raphy revealed complete bilateral ICA occlusion at the base of the skull with collateral supply from the posterior circulation (Figure 3). Cerebral artery bypass surgery was not performed as collateral circulation had already been developed per se. Hormonal profile was unchanged. Takayasu's disease was excluded as ultrasound of the heart, triplex of the vessels of the legs and computed angiography of cervical, thorax and abdomen were normal. Markers of thrombophilia were negative as well. LYH was considered to be the most possible cause of bilateral occlusion of both ICAs. The dose of methylprednisolone was escalated and anticoagulant treatment was added.

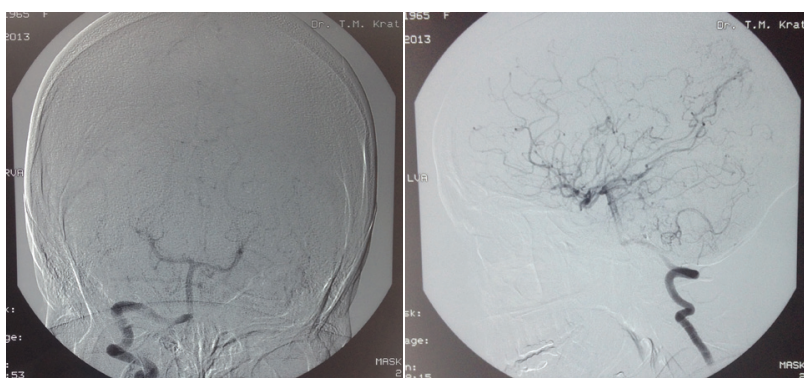

Figure 3. a) Computed tomography angiography demonstrated complete bilateral ICA occlusion at the base of the skull. b) Collateral supply from the posterior circulation is also shown. 


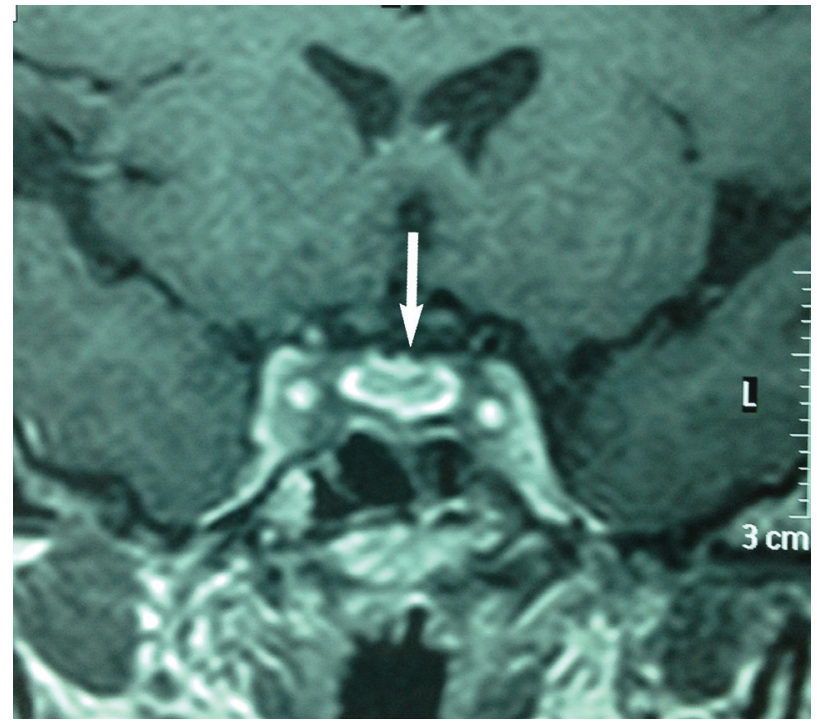

Figure 4. Pituitary T1-weighed coronal MRI scan three months after azathioprine treatment. Excessive reduction of the pituitary mass and insignificant extension at the cavernous sinuses is shown.

A month later, the patient developed Cushing's phenotype (buffalo hump, hirsutism and abdominal obesity) as well as diabetes mellitus and resistant hypertension. As the attempt to gradually reduce glucocorticoid dosage resulted in a relapse of the disease, an alternative option of immunosuppressive therapy with azathioprine was considered. Therapy was initiated at a dose of $100 \mathrm{mg}$ qd. A thorough explanation of the potential side effects was presented before obtaining the patient's consent. The new therapeutic regimen was well tolerated and 3 months later a considerable reduction of the pituitary mass with insignificant extension to the cavernous sinuses was observed (Figure 4). After 12 months of follow-up, the dose was reduced to $50 \mathrm{mg}$ qd and anterior pituitary function was restored, without any replacement therapy. Six months later, the treatment was discontinued and the patient now has no clinical or radiological evidence of the disease except for the permanent occlusion of both internal carotid arteries (Figure 5).

\section{DISCUSSION}

$\mathrm{PH}$ is a rare disorder involving inflammation of the pituitary gland that causes compression of anatomic structures and hypopituitarism. PH with extrapituitary spread to the surrounding structures is even rarer and requires prompt diagnosis and intervention. In this article, we report a very rare case of $\mathrm{PH}$ causing bilateral intracavernous carotid occlusion. Similar cases have been reported twice before. ${ }^{12,13}$

In the present report, the history and laboratory and imaging findings were consistent with $\mathrm{PH}$. In particular, the relatively rapid development of hypopituitarism, the thickened but intact pituitary stalk, the symmetrically enlarged pituitary gland with intense

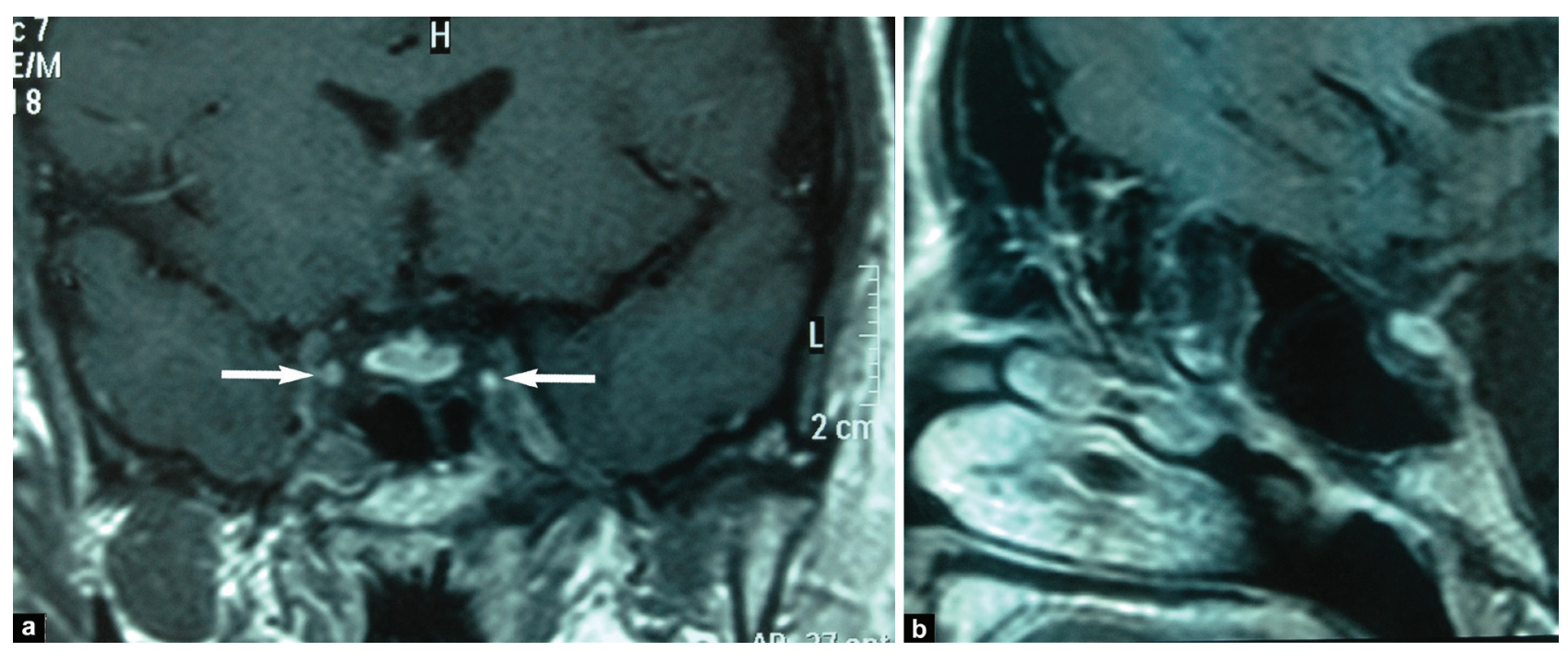

Figure 5. Pituitary coronal and sagittal T1-weighed MRI scan after gadolinium enhancement six months after the discontinuation of azathioprine treatment. No evidence of the disease was depicted apart from permanent occlusion of both ICAs (white arrows). 
enhancement following gadolinium, the presence of a dural tail and the extension of the pituitary lesion to the cavernous sinuses favoured the diagnosis of hypophysitis. After clinical workup to exclude secondary causes of the disease (sarcoidosis, Wegener granulomatosis, histiocytosis, tuberculosis, syphilis), $\mathrm{PH}$-and more specifically LYH- was suspected. Antipituitary antibodies were not measured in our patient as they are not routinely available in clinical practice and are considered neither sensitive nor specific markers of autoimmune pituitary disease..$^{10,11}$ Nevertheless, despite the lack of evidence of concomitant autoimmune disease, the response to pharmaceutical doses of corticosteroid treatment provided the rationale for the diagnosis of LYH in our patient.

Bilateral ICA occlusion associated with $\mathrm{PH}$ is an exceptionally rare clinical entity. To the best of our knowledge, only two cases of bilateral obstruction of ICAs in combination with aggressive forms of lymphocytic hypophysitis have been reported. ${ }^{12,13}$ Similarly to our case, recurrence of LYH after decreasing the corticosteroid dose and a prompt response after increasing the dose was described in the report of Melgar et al. ${ }^{12}$ However, no alternative treatment was tested and no further data of follow- up were provided. In our patient, earlier testing and diagnosis could probably have prevented bilateral occlusion of the ICAs and the subsequent stroke.

The underlying mechanism of artery occlusion during hypophysitis remains unclear. Probably, in the case of LYH, cavernous carotid occlusion is the result of a spread of the inflammatory process into the wall of the vessels in this area, causing the complete occlusion of the arteries. Apart from the inflammatory process, infiltration by high levels of IgG4 positive plasma cells (IgG4-related mechanisms) may also be involved.

Autoimmune arteritis has been reported to coexistence with granulomatous hypophysitis. ${ }^{14}$ However, this entity was excluded in our patient. In addition, other severe conditions such as fungal and bacterial infections have been described as causing bilateral ICA occlusion. ${ }^{15,16}$ However, these conditions are observed in immunosuppressed patients and evolve rapidly into fatal carotid thrombosis. They were thus also excluded.
As already mentioned, high doses of glucocorticoids remain the treatment of choice. They should be used in symptomatic cases such as in our patient, although asymptomatic cases are also frequently observed. Our patient was initially treated with high doses of glucocorticoids resulting in improvement of clinical symptoms and imaging findings. However, the relapse of $\mathrm{PH}$ following the tapering attempt with glucocorticoids and the development of iatrogenic Cushing's syndrome prompted selection of azathioprine as an alternative therapy option. Because of cavernous sinuses involvement, surgery was not indicated in our patient as an alternative choice of treatment.

Azathioprine is an imidazolyl derivative of 6-mercaptopurine used as an immunosuppressive agent in various conditions such as lupus, rheumatoid arthritis and Crohn's disease. It antagonizes purine metabolism and can affect rapidly growing cells by inhibiting synthesis of DNA, RNA and proteins. ${ }^{17}$ Our patient tolerated well the azathioprine treatment, with documented improvement in pituitary imaging, apart from the complete occlusion of both ICAs. Although the relevant literature is limited, azathioprine treatment could be a safe alternative treatment for $\mathrm{PH}$ when glucocorticoids are discontinued due to their side effects. In a few cases of aggressive LYH, azathioprine was equally effective in reducing pituitary mass. ${ }^{18}$ Our patient is stable with no signs of disease recurrence one year after discontinuation of the treatment.

Similarly to our report, in the two other cases with ICA involvement, the administration of glucocorticoids was effective in reducing pituitary mass but not as effective in regressing ICAs occlusion. ${ }^{11,12}$ Other alternative therapies were not needed since no adverse effects were observed with corticosteroids.

Finally, it should be mentioned that no artery bypass surgery was needed in our patient in contrast to previously reported cases. ${ }^{11}$ Intracranial circulation depends on collateral circulation by the vertebrobasilar axis which, in our patient, developed due to chronic progressive invasion by the inflammatory process to both carotid arteries.

The natural course of $\mathrm{PH}$ remains unpredictable. It is usually a benign condition with a good response to treatment. Sometimes, if left untreated, the final outcome is an empty sella. ${ }^{19}$ In rare cases, bilateral 
ICA occlusion may develop in the course of LYH with cavernous sinuses involvement, as observed in our patient, causing serious complications. Prompt identification of artery occlusion in the course of hypophysitis is critical and should lead to optimization of medical management, thereby preventing deterioration and avoiding stroke or other grave consequences. The efficacy of azathioprine treatment in complicated cases of $\mathrm{PH}$ suggests that it should be considered as an alternative regimen when glucocorticoids are contraindicated or are associated with serious adverse events.

\section{DISCLOSURE SUMMARY}

The authors have nothing to disclose.

\section{REFERENCES}

1. Ahmed SR, Aiello DP, Page R, Hopper K, Towfighi J, Santen RJ, 1993 Necrotizing infundibulo-hypophysitis: a unique syndrome of diabetes insipidus and hypopituitarism. J Clin Endocrinol Metab 76: 1499-1504.

2. Hama S, Arita K, Tominaga A, et al, 1999 Symptomatic Rathke's cleft cyst coexisting with central diabetes insipidus and hypophysitis: case report. Endocr J 46: 187-192.

3. Cheung CC, Ezzat S, Smyth HS, Asa SL, 2001 The spectrum and significance of primary hypophysitis. J Clin Endocrinol Metab 86: 1048-1053.

4. Tashiro T, Sano T, Xu B, et al, 2002 Spectrum of different types of hypophysitis: a clinicopathologic study of hypophysitis in 31 cases. Endocr Pathol 13: 183-195.

5. Lecube A, Francisco G, Rodríguez D, et al, 2003 Lymphocytic hypophysitis successfully treated with azathioprine: first case report. J Neurol Neurosurg Psychiatry 74: 1581-1583.

6. Tubridy N, Saunders D, Thom M, 2001 Infundibulohypophysitis in a man presenting with diabetes insipidus and cavernous sinus involvement. J Neurol Neurosurg Psychiatry 71: 798-801.

7. Papanastasiou L, Pappa T, Tsiavos V, et al, 2011 Aza- thioprine as an alternative treatment in primary hypophysitis. Pituitary 14: 16-22.

8. Nishioka H, Akada K, Miki T, et al, 1994 A case of lymphocytic hypophysitis with massive fibrosis and the role of surgical intervention. Surg Neurol 42: 74-78.

9. Selch MT, DeSalles AAF, Kelly DF, et al, 2003 Stereotactic radiotherapy for the treatment of lymphocytic hypophysitis. J Neurosurg 99: 591-596.

10. Tanaka S, Tatsumi KI, Takano T, et al, 2003 Anti-alphaenolase antibodies in pituitary disease. Endocr J 50: 697-702.

11. De Bellis A, Ruocco G, Battaglia M, et al, 2008 Immunological and clinical aspects of lymphocytic hypophysitis. Clin Sci (Lond) 114: 413-421.

12. Melgar MA, Mariwalla N, Gloss DS, et al, 2006 Recurrent lymphocytic hypophysitis and bilateral intracavernous carotid artery occlusion. An observation and review of the literature. Neurol Res 28: 177-183.

13. Peruzzotti-Jametti L, Strambo D, Sangalli F, De Bellis A, Comi G, Sessa M, 2012 Bilateral Intracavernous Carotid Artery Occlusion Caused by Invasive Lymphocytic Hypophysitis. J Stroke Cerebrovasc Dis 21: 918

14. Toth M, Szabo P, Racz K, et al, 1996 Granulomatous hypophysitis associated with Takayasu's disease. Clin Endocrin 1996; 45: 499-503.

15. Anaissie EJ, Shikhai AH, 1985 Rhinocerebral mucormycosis with intimal carotid occlusion: Report of two cases and review of the literature. Laryngoscope 95: 1107-1113.

16. Galetta SI, Wulc AE, Goldberg HI, et al, 1990 Rhinocerebral mucormycosis: Management and survival after carotid occlusion. Ann Neurol 28: 103-107.

17. Armstrong VW, Oellerich M, 2001 New developments in the immunosuppressive drug monitoring of cyclosporine, tracolimus, and azathioprine. Clin Biochem 34: 9 .

18. Lecube A, Francisco G, Rodríguez D, et al, 2003 Lymphocytic hypophysitis successfully treated with azathioprine: first case report. J Neurol Neurosurg Psychiatry 74: 1581-1583.

19. Karaca Z, Tanriverdi F, Unluhizarci K, Kelestimur F, Donmez H, 2009 Empty sella may be the final outcome in lymphocytic hypophysitis. Endocr Research 34: 10-17. 